\title{
On the occurrence of thermal explosion in a reacting gas: the effects of natural convection and consumption of reactant
}

Ting-Yueh Liu, Alasdair N. Campbell, Allan N. Hayhurst and Silvana S. S. Cardoso* Department of Chemical Engineering and Biotechnology, University of Cambridge, Pembroke St, Cambridge, $U K, C B 23 R A$.

*Corresponding author: Fax +44(0)1223334796, sssc1@cam.ac.uk

\begin{abstract}
Whether or not a chemical reaction in a fluid leads to an explosion is shown to depend on four timescales: that for the chemical reaction to heat up the fluid containing the reactants and products, for heat conduction out of the reactor, for natural convection in the fluid, and finally for chemical reaction. This approach is developed for an irreversible, $n$-th order chemical reaction, $\mathrm{A} \rightarrow \mathrm{B}$ occurring exothermically in a closed spherical vessel, whose wall is held at a fixed temperature. These four timescales are expressed in terms of the physical and chemical parameters of the system. A new three-dimensional regime diagram is proposed, in which the three effects inhibiting explosion, viz. the consumption of reactant, and heat removal both by thermal conduction and by natural convection, appear separately. Numerical simulations are performed for laminar natural convection occurring, so that the development of temperature, composition and velocity throughout a reacting gas is computed for increasing times. The results are compared with previous experimental measurements in the gas phase for the decomposition of azomethane. The criterion for an explosion is considered in some detail; it appears that these systems explode if and when the maximum dimensionless rise in temperature exceeds a value close to 5 .
\end{abstract}

\section{Keywords}

Thermal explosions; natural convection; autoignition; conductive and convective heat transfer; consumption of reactant

\section{Nomenclature}

c Concentration of chemical species A

$c^{\prime} \quad$ Dimensionless concentration of A, $c^{\prime}=c / c_{0}$

$C_{p} \quad$ Specific heat at constant pressure

$C_{v} \quad$ Specific heat at constant volume

$D_{A} \quad$ Diffusion coefficient of species A

$E \quad$ Activation energy of reaction $\mathrm{A} \rightarrow \mathrm{B}$ 
$g \quad$ Acceleration due to gravity

$k_{0} \quad$ Rate constant of the reaction at $T=T_{0}$

$L \quad$ Characteristic length (radius) of the reactor

Le Lewis number $=\kappa / D_{A}$

$n \quad$ Order of reaction

$\underline{n} \quad$ Unit vector normal to the surface of the reactor

$p \quad$ Pressure

$p^{\prime} \quad$ Dimensionless pressure, $p^{\prime}=\left(p-p_{0}\right) / \rho_{0} U^{2}$

Pr Prandtl number

$q \quad$ Exothermicity of reaction $\mathrm{A} \rightarrow \mathrm{B}$

$\underline{r}$ Position vector

$\underline{r}^{\prime} \quad$ Dimensionless position vector, $\underline{r}^{\prime}=\underline{r} / L$

$R \quad$ Universal gas constant

$\mathrm{Ra} \quad$ Rayleigh number $=\beta g L^{3} \Delta T /(\kappa v)$

$S_{v} \quad$ Surface area per unit volume

$t \quad$ Time

$t^{\prime} \quad$ Dimensionless time, $t^{\prime}=U t / L$

$T \quad$ Temperature

$T_{0} \quad$ Constant wall temperature

$T^{\prime} \quad$ Dimensionless rise in temperature, $T^{\prime}=\left(T-T_{0}\right) / \Delta T_{s}$

$T_{a d}^{\prime} \quad$ Dimensionless adiabatic temperature rise, $T_{a d}^{\prime}=q c_{0} E /\left(\rho_{0} C_{p} R T_{0}^{2}\right)$

$\underline{u} \quad$ Velocity vector

$\underline{u}^{\prime} \quad$ Dimensionless velocity vector, $\underline{u}^{\prime}=\underline{u} / U$

$U \quad$ Scale for velocity

$Z \quad$ Pre-exponential factor in Arrhenius expression for $k$

$\beta \quad$ Coefficient of thermal expansion, $\beta=1 / T$

$\gamma \quad$ Ratio of specific heats, $\gamma=C_{p} / C_{v}$

$\delta \quad$ Frank-Kamenetskii number, $\delta=L^{2} k_{0} c_{0}{ }^{n} q E /\left(\kappa \rho_{0} C_{p} R T_{0}{ }^{2}\right)$

$\Delta T_{s} \quad$ Scale for temperature increase, $\Delta T_{s}=R T_{0}^{2} / E$

$\eta \quad \eta=\Delta T_{s} / T_{0}$

$\kappa \quad$ Thermal diffusivity

$v \quad$ Kinematic viscosity

$\rho_{0} \quad$ Density at $T=T_{0}$

$\tau_{C} \quad$ Timescale for convection

$\tau_{D} \quad$ Timescale for diffusion of heat

$\tau_{H} \quad$ Timescale for the exothermic reaction to heat the fluid by $\Delta T_{s}$

$\tau_{R} \quad$ Timescale for the depletion of reactant

$\chi \quad$ Heat-transfer coefficient

$\psi \quad$ Semenov number, $\psi=k_{0} c_{0}{ }^{n} q E /\left(\chi S_{v} R T_{0}{ }^{2}\right)$

\section{List of subscripts}

$0 \quad$ Properties at initial conditions

c Properties at the conductive limit under critical conditions

cr Properties at critical conditions

max Maximum 


\section{Introduction}

An exothermic chemical reaction proceeding in a gas contained in, say, a spherical vessel can end in an explosion; this is because the release of enthalpy results in the temperature of the reacting mixture increasing, with the consequence that the rates of both reaction and the production of heat continue to grow. However, an explosion is inhibited by heat being conducted to the walls of the containing vessel. Sometimes this loss of heat is augmented considerably by natural convection occurring in the reacting gas. The original works of Semenov [1] and Frank-Kamenetskii [2] considered, respectively, the two cases, in which: (a) convection is so vigorous that the temperature in the vessel is uniform; and (b) the transport of heat occurs by conduction only. For wellmixed systems, the reacting gas explodes when the Semenov number $\psi=k_{0} c_{0}{ }^{n} q E /\left(\chi S_{v} R T_{0}{ }^{2}\right)$ exceeds the critical value of $1 / \mathrm{e}$. When heat is transferred by thermal conduction alone, explosion occurs when the parameter (sometimes called the Frank-Kamenetskii number) $\delta=L^{2} k_{0} c_{0}{ }^{n} q E / \kappa \rho_{0} C_{p} R T_{0}{ }^{2}$ is greater than a critical value $\delta_{\text {cr. }}$. This critical value depends on the geometry of the system and has been calculated numerically for reactions without consumption of reactant [2] as 3.32 for a sphere, 2.0 for a cylinder and 0.88 for parallel plates; we note that these results are independent of the order of the reaction provided there is no consumption of reactant. However, in most reacting systems heat loss occurs due to the combined effects of natural convection and heat conduction. Recently, Liu et al. [3] proposed that in such systems the occurrence or not of an explosion depends on the relative magnitudes of three timescales: that for chemical reaction to heat up the fluid to ignition, the timescale for thermal conduction and that for natural convection. They summarized their results in a new two-dimensional regime diagram, in which Frank-Kamenetskii's purely conductive system and Semenov's well-mixed system appear as two limiting cases, represented by the two axes. The plane in between the two axes contains all the systems with different relative magnitudes of heat loss by conduction and by natural convection. This approach has the advantage of quantifying separately the stabilising effects of conduction and natural convection on an explosion.

Systems neglecting the consumption of reactant have been widely studied [1-7]. However, in addition to heat loss by conduction and convection, an explosion is inhibited by a drop in the rate of reaction, because one or more of the reactants inevitably disappears. The present work extends the original ideas of Liu et al. [3] 
to explore how the consumption of reactant alters the onset of a thermal explosion. We investigate whether the effect of the consumption of reactant can be accounted for by the ratio of the magnitudes of the timescale for the exothermic reaction to heat up the fluid to the ignition temperature and the characteristic timescale for chemical reaction. If this turns out to be the case, whether or not an exothermic chemical reaction leads to an explosion is determined entirely by four timescales, viz., those for heating up the system by the particular exothermic reaction, for cooling by thermal conduction, for natural convection and, finally, for the disappearance of reactant. This approach separates the three stabilising effects, i.e. of thermal conduction, natural convection and reactant being consumed. The theoretical and numerical work below considers an $n$-th order exothermic reaction in a gas or liquid contained in a spherical vessel with a constant wall temperature, when the consumption of the reactant is important. For a spherical vessel, natural convection has been shown $[8,9]$ to become significant above $\mathrm{Ra} \sim 500$.

\section{Theoretical analysis}

The reaction considered here is of $n$-th order and has the simplest mechanism of $\mathrm{A} \rightarrow \mathrm{B}$. Thus, a pure gaseous or liquid reactant undergoes an exothermic isomerisation inside a closed spherical vessel. The initial pressure and concentration of the reactant $\mathrm{A}$, are $p_{0}$ and $c_{0}$, respectively. The initial temperature of both the fluid and wall of the vessel is $T_{0}$. The radius of the vessel, $L$, is taken to be the characteristic length scale of the system. Following the approach first proposed by Cardoso et al. [10, 11], Liu et al. [3] have recently derived the fundamental equations describing this system, namely those for the conservation of reactant A, energy, momentum and mass. Using the Boussinesq approximation, these can be written [3] in non-dimensional form as:

$\frac{\partial c^{\prime}}{\partial t^{\prime}}+\underline{u}^{\prime} \cdot \nabla^{\prime} c^{\prime}=\frac{1}{\operatorname{Le}} \frac{\tau_{C}}{\tau_{D}} \nabla^{\prime 2} c^{\prime}-\frac{\tau_{C}}{\tau_{R}} \exp \left(\frac{T^{\prime}}{1+\eta T^{\prime}}\right) c^{\prime \prime}$

$\frac{1}{\gamma} \frac{\partial T^{\prime}}{\partial t^{\prime}}+\underline{u^{\prime}} \cdot \nabla^{\prime} T^{\prime}=\frac{\tau_{C}}{\tau_{D}} \nabla^{\prime 2} T^{\prime}+\frac{\tau_{C}}{\tau_{H}} \exp \left(\frac{T^{\prime}}{1+\eta T^{\prime}}\right) c^{\prime n}$

$\frac{\partial \underline{u^{\prime}}}{\partial t^{\prime}}+\underline{u}^{\prime} \cdot \nabla^{\prime} \underline{u}^{\prime}=-\nabla^{\prime} p^{\prime}+\operatorname{Pr} \frac{\tau_{C}}{\tau_{D}} \nabla^{\prime 2} \underline{u^{\prime}}-\frac{g}{g} T^{\prime}$

$\nabla^{\prime} \cdot \underline{u}^{\prime}=0$ 
where the concentration of reactant, the temperature, velocity, pressure, spatial position and time have been non-dimensionalised according to:

$c^{\prime}=\frac{c}{c_{0}} ; \quad T^{\prime}=\frac{T-T_{0}}{R T_{0}^{2} / E} ; \quad \underline{u}^{\prime}=\frac{\underline{u}}{U} ; \quad p^{\prime}=\frac{p-p_{0}}{\rho_{0} U^{2}} ; \quad \underline{r}^{\prime}=\frac{\underline{r}}{L} ; t^{\prime}=\frac{U t}{L} ;$

The scale for the velocity due to natural convection [3] is:

$U \sim\left(\beta g L R T_{0}^{2} / E\right)^{1 / 2}$

Here, $\beta$ is the coefficient of thermal expansion, $R$ is the universal gas constant and $E$ is the activation energy. Also, Le $=\kappa / D_{A}$ is the Lewis number and $\operatorname{Pr}=\nu / \kappa$ is the Prandtl number. The four timescales determining the behaviour of the system are: $\tau_{D}$, the time for thermal conduction; $\tau_{C}$, the timescale for fluid to move by natural convection over the characteristic distance $L$ in the vessel; $\tau_{H}$, the timescale for the exothermic reaction to heat up the fluid to the ignition temperature; and finally, $\tau_{R}$, the timescale for the depletion of reactant. These four timescales may be expressed in terms of the physical and chemical parameters of the system as $\tau_{D}=\frac{L^{2}}{\kappa} ; \tau_{C}=\frac{L}{U} ; \tau_{H}=\frac{\rho_{0} C_{p} R T_{0}^{2}}{k_{0} c_{0}{ }^{n} q E} ; \tau_{R}=\frac{1}{k_{0} c_{0}{ }^{n-1}}$

We note that $\tau_{R} / \tau_{H}=T_{a d}^{\prime}$ is the commonly used dimensionless adiabatic temperature rise, defined as $T_{a d}^{\prime}=\frac{q c_{0}}{\rho_{0} C_{p}} \frac{E}{R T_{0}^{2}}$

The boundary and initial conditions for Eqs (1) - (4) are as follows. Initially, the temperature and concentration of $\mathrm{A}$ in the reactor are spatially uniform at $T_{0}$ and $c_{0}$, respectively; also, the fluid is assumed to be at first motionless. The temperature of the wall is always held constant at $T_{0}$, so that heat is removed via the wall. The fluid velocity and the flux of chemical species are both always zero at the wall; this means that the effects of any heterogeneous reaction at the wall are ignored. These conditions can thus be stated nondimensionally as:

$\underline{u^{\prime}}=0 ; \underline{n} \cdot \nabla^{\prime} c^{\prime}=0 ; T^{\prime}=0$ at $r^{\prime}=1, \forall t^{\prime}$

$T^{\prime}=0 ; c^{\prime}=1 ; \underline{u}^{\prime}=0$ at $t^{\prime}=0,0 \leq r^{\prime} \leq 1$

where $\underline{n}$ is a unit vector normal to the surface of the vessel and $r^{\prime}$ denotes the dimensionless radial position. 
Eqs. (1) - (4) and (9) - (10) show that there are eight dimensionless groups determining the evolution of the dimensionless concentration, temperature and velocity in the vessel; they are:

$$
\frac{\tau_{H}}{\tau_{D}} ; \frac{\tau_{H}}{\tau_{C}} ; \frac{\tau_{H}}{\tau_{R}} ; \text { Le } ; \eta=\frac{R T_{0}}{E} ; \operatorname{Pr} ; \gamma=\frac{C_{p}}{C_{v}} ; n
$$

For a specified reaction and initial temperature, $T_{0}$, the groups $\eta, \gamma, n, \operatorname{Pr}$ and Le are fixed. Therefore the behaviour of the system is determined simply by the three ratios $\left(\tau_{H} / \tau_{D}\right),\left(\tau_{H} / \tau_{C}\right)$ and $\left(\tau_{H} / \tau_{R}\right)$. This important conclusion is exploited below.

\section{Regime diagram for explosion}

It has just been demonstrated that the behaviour of the system can be depicted on a three-dimensional regime diagram, as sketched in Fig. 1, where the three ratios of timescales $(11 \mathrm{a}-\mathrm{c})$ appear as coordinates. In this diagram there should be a surface separating the space into two regions: one near the origin, where explosions occur and another farther from the origin where the stabilising effects of heat conduction, natural convection and consumption of reactant are sufficient for explosions to be prevented. The vertical axis for $\left(\tau_{H} / \tau_{D}\right)$ represents the purely conductive limit ignoring depletion of reactant, i.e., the systems considered by

Frank-Kamenetskii [2]. Otherwise, the axis for $\left(\tau_{H} / \tau_{C}\right)$ represents the well-mixed limit with no consumption of reactant, where the assumptions of Semenov [1] apply. The third axis, for $\left(\tau_{H} / \tau_{R}\right)$, represents a measure of the effect of the disappearance of reactant on the heating up of the fluid; for example, if the chemical reaction in effect depletes the reactant much faster than fluid is heating up (e.g., because the heat of reaction is very small), then $\tau_{R}<<\tau_{H}$, and we expect the temperature rise in the fluid to be small and explosion not to occur. The regime diagram in Fig. 1 has the important advantage of showing separately the effects of cooling by thermal conduction, of cooling by natural convection, and of the depletion of reactant, on the transition from stable to explosive conditions.

\subsection{Systems with negligible consumption of reactant}

The plane defined by the axes $\left(\tau_{H} / \tau_{D}\right)$ and $\left(\tau_{H} / \tau_{C}\right)$ is shown in Fig. 2 and refers to systems with diffusion, convection and reaction, but without any significant consumption of reactant. Systems on this plane have been widely investigated [12-18] and results have been reported as an increase in the magnitude of the 
critical Frank-Kamenetskii number with Rayleigh number. More recently, this plane was studied in detail by Liu et al. [3], who demonstrated that the conditions for neglecting the consumption of reactant are $\left(\tau_{H} / \tau_{C}\right)>>$ $\left(\tau_{H} / \tau_{R}\right)$ and $\left(\tau_{H} / \tau_{D}\right) \gg>$ Le $\left(\tau_{H} / \tau_{R}\right)$. Also, it should be recalled [10,11] that the relative importance of thermal conduction and natural convection in a system in Fig. 2 is indicated by the Rayleigh number:

$\mathrm{Ra}=\left(\frac{\tau_{D}}{\tau_{C}}\right)^{2} \operatorname{Pr}^{-1}=\left(\frac{\tau_{H}}{\tau_{C}}\right)^{2}\left(\frac{\tau_{H}}{\tau_{D}}\right)^{-2} \operatorname{Pr}^{-1}$.

Thus, for a fixed $\left(\tau_{H} / \tau_{R}\right)$, a straight line through $\left(\tau_{H} / \tau_{D}\right)=\left(\tau_{H} / \tau_{C}\right)=0$ has a slope of $\sqrt{ }(\operatorname{RaPr})$. Such a straight line is accordingly the locus of constant $\mathrm{Ra}$. When $\mathrm{Ra}<500$, heat transfer in the fluid is controlled by conduction $[8,9]$. In the range $500<\mathrm{Ra}<10^{6}$, laminar convection dominates heat transfer. For $\mathrm{Ra}>10^{6}$, the flow is turbulent $[10,19]$.

Importantly, the timescales defined in $(7 \mathrm{a}-\mathrm{d})$ can be expressed in terms of the Frank-Kamenetskii and Rayleigh numbers as:

$$
\frac{\tau_{H}}{\tau_{D}}=\frac{1}{\delta} ; \frac{\tau_{H}}{\tau_{C}}=\frac{1}{\delta}(\operatorname{RaPr})^{1 / 2}
$$

Eq. (13a) can be used to identify the expected value of $\left(\tau_{H} / \tau_{D}\right)$ for the transition to explosion in the conductive limit, when $\left(\tau_{H} / \tau_{D}\right)_{c}=1 / \delta_{c}=1 / 3.32=0.30$, where $\left(\tau_{H} / \tau_{D}\right)_{c}$ is the critical value of $\left(\tau_{H} / \tau_{D}\right)$ when heat transfer is purely conductive. This implies that explosion will occur if the timescale for heating the fluid to the ignition temperature owing to the exothermicity of the reaction is less than $\sim 1 / 3$ of the timescale for conduction of heat. This critical value is shown in Fig. 2 as the approximately horizontal boundary between explosive and non-explosive behaviours for $\mathrm{Ra}<200$ and $\left(\tau_{H} / \tau_{R}\right)=0$. For $\mathrm{Ra}>200$, convection becomes increasingly important and the critical value of $\left(\tau_{H} / \tau_{D}\right)$ decreases sharply; at Ra 500, the critical value of $\left(\tau_{H} / \tau_{D}\right)$ is approximately $20 \%$ smaller than in Frank-Kamenetskii's conductive limit and transport of heat by convection has become significant. For Ra in the range $500<\mathrm{Ra}<10^{6}$, Liu et al. [3] conducted numerical simulations and identified the transition between stable and explosive behaviour; this explosion boundary is shown in Fig 2. When $\mathrm{Ra}>10^{6}$, natural convection is vigorous and turbulent $[19,20]$; then the system approximates to the well-mixed (Semenov) case [1]. However, conduction of heat still plays a role in the thin 
thermal boundary layer adjacent to the reactor wall. Liu et al. [3] derived an expression for the transition from an explosion to non-explosive reaction in this turbulent region, which is shown as the dotted line in Fig. 2. In summary, for $\left(\tau_{H} / \tau_{R}\right)=0$ the transition from stable to explosive behaviour for an exothermic reaction of order $n=1.4$, with negligible consumption of reactant and for spherical geometry, can be approximated by:

$$
\frac{\tau_{H}}{\tau_{D}}=\left\{\begin{array}{cc}
0.30 & R a<500 \\
0.86\left(\tau_{H} / \tau_{C}\right)^{-0.74} & 500<R a<10^{6} \\
1.4 \times 10^{2}\left(\tau_{H} / \tau_{C}\right)^{-2} & R a>10^{6}
\end{array}\right.
$$

for $\eta=0.027, \operatorname{Pr}=\operatorname{Le}=1$ and $\gamma=1.018$. We note here that the order of the reaction and the values of $\eta$ and $\gamma$ in these simulations have been chosen to represent the thermal decomposition of azomethane [21] for which experimental results will be presented in section 5 .

\subsection{Systems with consumption of reactant}

In Fig. 1, the vertical plane defined by the axes $\left(\tau_{H} / \tau_{R}\right)$ and $\left(\tau_{H} / \tau_{D}\right)$ contains purely conductive systems with depletion of reactant, but without natural convection. Systems on this plane have been studied before [22-26]. Fig. 3 presents this plane, but for a first-order reaction only. The analytical predictions of the explosion boundary by Thomas [22] and Boddington et al. [27], as well as the numerical results of Adler and Enig [23] and Tyler and Wesley [25], are shown. Also presented are the experimental observations of Griffiths and Mullins [26] for the decomposition of di-t-butyl peroxide in the gas phase. The analytical results of Thomas [22] are approximate, because the spatial temperature distribution was described by only the maximum temperature at the centre of the vessel and an effective heat transfer coefficient. In addition, the conservation equation for the reactant was solved by neglecting the effect of temperature on the reaction rate. Solutions to these approximate equations were obtained in the limit of a large adiabatic temperature rise. Boddington et al. [27] carried out an elegant asymptotic analysis, in which the spatial distributions of the temperature and concentration were taken into account. Their calculations also assumed a large adiabatic temperature rise; their solution shown in Fig. 3 is for an infinite Biot number, i.e. for the external resistance to heat transfer being negligible. For the numerical results of Adler and Enig [23] the fluid's temperature distribution was approximated in a similar way to that by Thomas [22]. Tyler and Wesley [25] conducted a full numerical simulation of the conservation equations for energy and chemical species for the decomposition of azomethane, 
which they assumed to be first order. Their results are fairly close to those of Thomas [22] for low consumptions of reactant, suggesting that the multiple simplifications in the latter model compensate for one another. Strangely, the experimental measurements of Griffiths and Mullins [26] lie well below the numerical predictions, particularly for the smaller values of $\left(\tau_{H} / \tau_{D}\right)$. The Rayleigh number in these experiments varied from $\sim 7$ to $\sim 700$, so it is possible that natural convection had some effect when $\mathrm{Ra}>200$, albeit small. The experimental results suggest an almost infinite slope for the dependence of $\left(\tau_{H} / \tau_{D}\right)$ on $\left(\tau_{H} / \tau_{R}\right)$, i.e. the rate of heat release decreases infinitely fast with reactant consumption, which is physically impossible, so it is likely that there were some other unidentified problems.

In order to understand the behaviour of the system on a horizontal plane of the regime diagram in Fig. 1 defined by the axes $\left(\tau_{H} / \tau_{C}\right)$ and $\left(\tau_{H} / \tau_{R}\right)$, numerical simulations were carried out. These are described next.

\section{Numerical method}

In the numerical simulations, $\left(\tau_{H} / \tau_{D}\right),\left(\tau_{H} / \tau_{C}\right)$ and $\left(\tau_{H} / \tau_{R}\right)$ were varied by changing the values of $\kappa, g$ and $q$, whilst the other groups in Eqs. (11d-h) remained constant. Simulations were carried out for laminar flow, i.e. for Ra $<10^{6}$. Eqs. (1) - (4) and (9) - (10) were solved numerically for a spherical reactor using the partial differential equation solver Fastflo [28], which uses the finite element method. The algorithm used was the same as described before [10]. The reaction analysed was the thermal decomposition of pure azomethane. The following parameters were chosen to match those used in previous experimental work by Archer [21]. Thus the radius of the vessel was $L=0.064 \mathrm{~m}$, the temperature of the wall was constant at $T_{0}=636.2 \mathrm{~K}$, the initial concentration of reactant was $c_{0}=0.37 \mathrm{~mol} \mathrm{~m}^{-3}$ (corresponding to $p_{0} \sim 0.02$ bar) and the order of reaction was $n=1.4$. The physico-chemical properties employed were: $C_{p}=2250 \mathrm{~J} \mathrm{~kg}^{-1} \mathrm{~K}^{-1}, Z=1.24 \times 10^{14} \mathrm{~mol}^{-0.4} \mathrm{~m}^{1.2}$ $\mathrm{s}^{-1}, E / R=23280 \mathrm{~K}$ (so $E=193.6 \mathrm{~kJ} \mathrm{~mol}^{-1}$ ) and $\gamma=1.018$. For simplicity it was further assumed that $\operatorname{Pr}=\mathrm{Le}=$ 1. The last assumption indicates $\kappa=v=D_{A}$, implying that the diffusivities for the transfer of heat, momentum and chemical species are equal. This is approximately true for gases, but not for liquids, for which $\operatorname{Pr}$ is significantly larger than unity.

\section{Results and Discussion}

\subsection{Evolution of axial temperature and concentration}


Figs. 4a(i) - (iv) plot the evolution (in dimensionless time) of the calculated dimensionless increase in temperature for three reactions with different values of $\left(\tau_{H} / \tau_{R}\right)$, but $\left(\tau_{H} / \tau_{D}\right)$ fixed. Figs. $4 \mathrm{a}(\mathrm{i})$ and (ii) show the rise in temperature at the centre and near the top of the vessel $(0.8 L$ above the centre $)$, respectively, at $\mathrm{Ra} \sim$ 500. So natural convection is not a dominant factor and thermal conduction determines the removal of heat at this small Ra, ignition is expected to occur near the centre of the vessel. It appears from Figs. 4a(i) and (ii) that initially the temperature increases at the same rate, regardless of the magnitude of $\left(\tau_{H} / \tau_{R}\right)$, i.e. the temperature increase is initially independent of $\left(\tau_{H} / \tau_{R}\right)$. This behaviour is expected at times $t<<\tau_{R}$, when the consumption of reactant has only a small effect. The plots for the lowest $\left(\tau_{H} / \tau_{R}\right)=0.0183$ end apparently prematurely; this is because for such an explosive reaction, the temperature increases so rapidly that numerical errors occur when the finite element method is used. Otherwise, $T^{\prime}$ rises to values as high as $\sim 10$ for the two larger $\left(\tau_{H} / \tau_{R}\right)$. In fact, after a slow rise the increase in temperature accelerates, suggesting an explosion. Also for a larger $\left(\tau_{H} / \tau_{R}\right)$ the maximum $T^{\prime}$ is smaller.

Figs. $4 \mathrm{a}(\mathrm{iii})$ and (iv) show the behaviour for the same values of $\left(\tau_{H} / \tau_{D}\right)$ and $\left(\tau_{H} / \tau_{R}\right)$ as in Figs. $4 \mathrm{a}(\mathrm{i})$ and (ii), but at a much higher $\mathrm{Ra} \sim 10^{5}$. Cooling of the fluid at such a high $\mathrm{Ra}$ is enhanced by natural convection. Consequently the maximum temperature is significantly lower than in Figs. 4a(i) and (ii), when natural convection is absent; also the maximum temperature is reached over a much longer timescale. In fact, Figs. 4a(iii) and (iv) indicate that reaction is stable, i.e. not explosive. However, the three reactions with different $\left(\tau_{H} / \tau_{R}\right)$ again initially heat up at approximately the same rate, but the maximum temperature is now observed near the top of the vessel, due to the hot upwards flow at the centre of the vessel, caused by the difference in temperature between the hotter centre and the colder wall. The oscillations in the temperature early in the reaction, whilst the system heats up to a pseudo-steady state, are associated with instabilities in the developing flow field and will be discussed below.

Figs. 4b(i) - (iv) show dimensionless plots of concentration of reactant against time, corresponding to the cases shown in Figs. 4a(i) - (iv). Thus Figs. 4b(i) and (ii) are for a low Ra with negligible natural convection. They again refer, respectively, to the centre of the reactor and near the top of the vessel (at $0.8 \mathrm{~L}$ directly above the centre). In Fig. 4b(i) and (ii), the concentration of reactant drops dramatically (with 
$\left.\partial^{2} c^{\prime} / \partial t^{\prime 2}<<0\right)$, when the sudden large increase in temperature, i.e. explosion, occurs, as seen in Figs. 4a(i) and (ii). In fact, only $10 \%$ of the reactant has been consumed locally when explosion occurs for $\left(\tau_{H} / \tau_{R}\right)=0.0183$ in Fig. 4 b(i). However, in the case of the two larger $\left(\tau_{H} / \tau_{R}\right)$ in Fig. 4b(ii), explosion occurs when the local concentration of reactant has fallen by over $50 \%$. Such a picture contrasts with Figs. 4b(iii) and (iv), which show less depletion of reactant when Ra is large. In fact, in Fig. 4b(iv), the local conversion of reactant when $T^{\prime}$ reaches a maximum of $\sim 1.2$ is only $\sim 5 \%$ for the case with $\left(\tau_{H} / \tau_{R}\right)=0.0183$, and $\sim 20 \%$ for reaching $T_{\max }^{\prime} \sim$ 1.05 for the larger $\left(\tau_{H} / \tau_{R}\right)$ of 0.1136 , i.e. showing an even larger consumption of reactant on reaching a lower $T_{\max }^{\prime}$. It is now clear that reaction proceeds non-explosively in Fig. 4(iii) and (iv), with the concentration decreasing fairly slowly with time, but at a slightly higher rate near the top of the vessel than at the centre, due to the higher local temperature.

Figs. 4c(i) - (iv) show the corresponding dimensionless plots of speed (again at the centre of the vessel or just below its top) against time. The characteristic speed $(U)$ has a different magnitude for each of the curves. Figs. $4 \mathrm{c}(\mathrm{i})$ and (ii) are for $\left(\tau_{H} / \tau_{C}\right) \sim 2.02$, i.e. $\mathrm{Ra} \sim 500$, indicating negligible fluid motion due to natural convection; hence $U$ is small. In fact, $U \sim 0.076 \mathrm{~m} \mathrm{~s}^{-1}, 0.016 \mathrm{~m} \mathrm{~s}^{-1}$ and $0.012 \mathrm{~m} \mathrm{~s}^{-1}$ for the cases with $\left(\tau_{H} / \tau_{R}\right)=0.0183,0.0842$ and 0.1136 , respectively. On the other hand, Figs. $4 \mathrm{c}$ (iii) and (iv) show the speeds for $\left(\tau_{H} / \tau_{C}\right) \sim 28.9\left(\mathrm{Ra} \sim 10^{5}\right)$, where the fluid flows strongly and the values of $U$, for $\left(\tau_{H} / \tau_{R}\right)$ being 0.0183 , 0.0842 and 0.1136 , are $1.070 \mathrm{~m} \mathrm{~s}^{-1}, 0.233 \mathrm{~m} \mathrm{~s}^{-1}$ and $0.177 \mathrm{~m} \mathrm{~s}^{-1}$, respectively. It is thus clear that again $U$ is significantly lower for a higher $\left(\tau_{H} / \tau_{R}\right)$. In Fig. 4c(iii), the speed of the flow shows a few cyclic fluctuations with decreasing amplitudes, before damping out after $t^{\prime} \sim 45$. These fluctuations coincide with the those in temperature shown in Fig. 4a(iii), suggesting that these fluctuating temperatures are due to the unstable flow field. However, as the fluid flow progressively stabilises, the temperature reaches its pseudo-steady value $\left(t^{\prime} \sim\right.$ 45) more smoothly. This is because whilst the reaction proceeds, the consumption of reactant reduces the generation of heat, slows the rate of increase in temperature and so provides a weaker driving force for natural convection. Consequently the fluctuations are gradually weakened and eventually die out. Similar phenomena can be observed in Fig. 4c(iv) and its corresponding plot of temperature (Fig. 4a(iv)), for near the top of the vessel. We note that similar fluctuations have been observed in the experiments by Archer involving the 
thermal decomposition of azomethane [21] and have also been recorded during the heating of an inert system [29].

\subsection{Evolution of the fields for temperature, concentration, flow and speed}

Figs. 5(a) and (b) show the computed evolution of: (i) temperature, (ii) concentration of reactant, (iii) flow field and (iv) the speed of the flow, for $\left(\tau_{H} / \tau_{C}\right)=2.02(\mathrm{Ra} \sim 500)$ and $\left(\tau_{H} / \tau_{C}\right)=29.47\left(\operatorname{Ra} \sim 10^{5}\right)$, respectively. Throughout Fig. $5\left(\tau_{H} / \tau_{R}\right)=0.1136$ and $\left(\tau_{H} / \tau_{D}\right)=0.09$. In Fig. 5(a), a sudden increase in temperature at the centre of the vessel occurs between $t^{\prime}=3.1$ and 3.3, when the local concentration of reactant has decreased by $95 \%$, indicating an ignition. As expected for reaction at such a low $\left(\tau_{H} / \tau_{C}\right)=2.02$, the location of the highest temperature remains close to the vessel's centre. The concentration field for the reactant is also symmetric about the vertical axis with the position of the lowest local concentration of reactant, corresponding to the hottest spot, slightly above the centre. The motion of the fluid inside the vessel appears to be insignificant, since the maximum dimensionless speed in the central upwards flow is $\left|\underline{u}^{\prime}\right|=0.587$, giving a maximum speed of only $7.1 \mathrm{~mm} \mathrm{~s}^{-1}$. By contrast, in Fig. 5(b) the reaction is clearly non-explosive, with the highest $T^{\prime}$ achieved being only $\sim 1.02$. The spatial variation in temperature shows a distorted profile with the hottest spot near the top of the vessel - in fact, near the bottom of the reactor the temperature hardly rises. The clear stratification of temperature indicates a relatively strong fluid flow, which brings heat from the centre towards the top of the vessel. Subsequently the reacting fluid cools, whilst moving downwards fairly close to the colder wall. The distribution of reactant is now much more uniform than in Fig. 5(a), no doubt because of the enhanced mixing caused by the vigorous flow. The fluid reaches a maximum dimensionless speed of $\left|\underline{u^{\prime}}\right| \sim$ 0.184 , corresponding to a speed of $0.0326 \mathrm{~m} \mathrm{~s}^{-1}$, when flowing downwards near the wall $(0.9 L$ from the centre on the equator) at $t^{\prime} \sim 13.3$; then the ratio of the speed at the centre to that near the wall is $\sim 0.89$, which is the closest to unity throughout the entire reaction. Significant fluid motion appears after $t^{\prime} \sim 7.5$ and by the time $t^{\prime} \sim$ 15.8 the flow field has in effect developed fully, when appreciable gradients in the temperature appear in the bulk of the vessel. Whilst changes in the speed of the flow become very small, the ratio of the speed at the centre to that at $0.1 L$ away from the wall on the equator is maintained at $\sim 0.5$; during this time the temperature gradients continue to build up in the top half of the vessel. The fluid in the blue region between the upwards 
flow at the centre and the downwards flow at the wall is almost stagnant, corresponding to the eye of a vortex, symmetric around the vertical axis.

\subsection{Criterion for an explosion}

In the cases described above, a fairly clear distinction could usually be drawn between explosive and non-explosive cases. However, for the purposes of locating a region in Fig. 1 where explosions occur, a clear quantitative criterion for an explosion is necessary. For a system with no consumption of reactant, an explosion can be straightforwardly identified by the disappearance of a steady state. Such an analysis was used by FrankKamenetskii [2] to define $\delta_{\text {cr. }}$ He also showed [2] that for a zeroth-order reaction (or no loss of reactant) with only thermal conduction in a spherical reactor, the system explodes if $T^{\prime}$ exceeds 1.6. Otherwise, when the consumption of reactant is considered, the definition of an explosion used by Frank-Kamenetskii ceases to be appropriate, because the temperature rise will always remain bounded. Many different approaches to defining explosions have been adopted for such cases. Rice et al. [30] carried out numerical studies of a well-mixed reactor and defined explosive conditions as those where an upward inflection in the plots of $T$ versus $t$ is observed. The ignition delay for a first-order reaction was arbitrarily defined as the time taken for the temperature to rise to the critical value of $T^{\prime}=2$. Such a critical value of $T^{\prime}$ is consistent with FrankKamenetskii's expression [31] for the maximum rise in temperature in a stable, well-mixed system; it has also been used subsequently by others $[22,32]$ as a general criterion for a first-order reaction leading to an explosion, when the reactant disappears to a significant extent.

The effect of the loss of reactant on criticality has been investigated in other ways. Thus FrankKamenetskii [31] employed an asymptotic analysis to develop an analytical expression incorporating the consumption of reactant in a well-mixed reactor. He did not explicitly state a criterion for explosion, but instead expanded the expression for $\delta_{\mathrm{cr}}$ to include the effects of the consumption of reactant on the explosion limits. This study was the first to highlight the important roles of $T_{a d}^{\prime}\left(i . e . \tau_{H} / \tau_{R}\right)$ and $n$. Thomas [22] performed a similar analysis and showed that the effect of reactant being consumed on criticality was approximately twice that derived by Frank-Kamenetskii [31]. This apparent discrepancy was explained by Gray and Lee [33], who identified an inconsistency in Frank-Kamenetskii’s original analysis [31]. Gray and Lee [33] also demonstrated that Frank-Kamenetskii's corrected result was consistent with Thomas’s [22]. 
A different approach to determine criticality was used by Adler and Enig [23], again for a well-stirred reactor with reactant being consumed. They examined the behaviour in $T^{\prime}$ versus $c^{\prime}$ space, rather than $T^{\prime}$ versus $t^{\prime}$. Criticality was defined as the existence of an inflection in the plot of $T^{\prime}$ against $c^{\prime}$. Adler and Enig [23] further suggested that the rise in temperature under critical conditions is $T^{\prime \prime}=1+n^{1 / 2}$ for an $n$-th-order reaction, indicating that $T^{\prime}=2$ for a first-order reaction to yield an explosion. However, Tyler and Wesley [25] investigated systems in which the temperature varied spatially due to thermal conduction. They defined an explosion by investigating the sensitivity of the maximum temperature rise, $T_{\max }^{\prime}$, to changes in $\delta$. The critical value of $\delta$ was arbitrarily defined as the point where a $1 \%$ increase in $\delta$ results in a doubling of $T_{\max }^{\prime}$. Tyler and Wesley [25] also showed that for progressively smaller values of $T_{a d}^{\prime}$, the transition from a stable to an explosive system becomes increasingly gradual. Consequently, the condition that $T^{\prime}=2$ for a first-order reaction exploding may no longer be appropriate. This assertion has been supported by Steensma and Westerterp [34], who suggested a value of $T^{\prime}=5$ to define an explosion, rather than $T^{\prime}=2$.

Boddington et al. [35] used parametric sensitivity to determine explosion limits in their study of Semenov's assumptions and model. They sought the value of $\psi$ for which $\partial T_{\max }^{\prime} / \partial \psi$ was at its maximum. In reality, this corresponds to the existence of an inflection in the plot of $T_{\max }^{\prime}$ versus $\psi$. Morbidelli and Varma [36] carried out a similar analysis, but instead of studying the sensitivity coefficient $\partial T_{\max }^{\prime} / \partial \psi$, they used the normalised sensitivity coefficient ( $\equiv \partial \ln T_{\max }^{\prime} / \partial \ln \psi$ ) to define the critical value of $\psi$. In this study, a simple investigation of the sensitivity of $T_{\max }^{\prime}$ to $\left(\tau_{H} / \tau_{C}\right)$ was performed for reactions with $\left(\tau_{H} / \tau_{D}\right)=0.125$ or 0.09 , for which plots of $T_{\max }^{\prime}$ against $\left(\tau_{H} / \tau_{C}\right)$ are shown in Fig. 6 for a range of $\left(\tau_{H} / \tau_{R}\right)$. It can be seen that when the timescale for reaction is much larger than that for heating up the system, e.g. $\tau_{H} \leq 0.05 \tau_{R}$, the plots of $T_{\max }^{\prime}$ versus $\left(\tau_{H} / \tau_{C}\right)$ become almost vertical, i.e. they are similar to the ideal case with no consumption of reactant. It is clear that for both values of $\left(\tau_{H} / \tau_{D}\right), T_{\max }^{\prime}=2$ appears to be the maximum rise in temperature under stable conditions, provided $\left(\tau_{H} / \tau_{C}\right)$ exceeds a particular value. When $\left(\tau_{H} / \tau_{C}\right)$ becomes only slightly smaller than this value for a given $\left(\tau_{H} / \tau_{D}\right), T_{\max }^{\prime}$ can exhibit a large, sudden jump in Fig. 6 and go towards the 
adiabatic temperature rise. However, at the higher $\left(\tau_{H} / \tau_{R}\right)$ in Fig. 6, the maximum values of $T_{\max }^{\prime}$ are much lower and the transition from the low to the high temperature regimes is more gradual, when $\left(\tau_{H} / \tau_{C}\right)$ is reduced. Eventually, $\left(\tau_{H} / \tau_{R}\right)$ becomes too large for an explosion to occur, e.g. when $\left(\tau_{H} / \tau_{R}\right)=0.1515$ in Fig. 6(a)), $T_{\max }^{\prime}$ only reaches $\sim 2.5$. The critical value of $\left(\tau_{H} / \tau_{C}\right)$ for a given $\left(\tau_{H} / \tau_{D}\right)$ and $\left(\tau_{H} / \tau_{R}\right)$ could be defined as the point where the maximum sensitivity of $T_{\max }^{\prime}$ to $\left(\tau_{H} / \tau_{C}\right)$ is observed, i.e. $\partial^{2} T_{\max }^{\prime} / \partial\left(\tau_{H} / \tau_{C}\right)^{2}=0$ at the point of inflection. The dashed-dotted line in Fig. 6 shows that the value of $T_{\max }^{\prime}$ at which this inflection occurs is a function of both $\left(\tau_{H} / \tau_{C}\right)$ and $\left(\tau_{H} / \tau_{R}\right)$. On exceeding the inflection point, $T_{\max }^{\prime}$ tends to the value of $T_{a d}^{\prime}$, which can be estimated from $T_{a d}^{\prime}=1 /\left(\tau_{H} / \tau_{R}\right)$. As expected, the plots for $\left(\tau_{H} / \tau_{R}\right)=0.0909$ in both Figs. 6(a) and (b) have the same $T_{a d}^{\prime}$, but also approximately the same shape during the transition from the sub-critical to the supercritical condition. However, compared with the plot for $\left(\tau_{H} / \tau_{R}\right)=0.0909$ in Fig. 6(a), the one in Fig. 6(b) is shifted horizontally to a larger value of $\left(\tau_{H} / \tau_{C}\right)$, indicating that an increase in $\left(\tau_{H} / \tau_{C}\right)$ is required for the reaction to remain stable when the effect of thermal conduction is reduced by changing $\left(\tau_{H} / \tau_{D}\right)$ from 0.125 to 0.09 . Fig. 6 also shows that for every explosive case the value of $T_{\max }^{\prime}$ at the point of inflection exceeds 5 . This is higher than the value $\left(T_{\max }^{\prime}=2\right.$ ) previously used $[22,23,30,32]$ to identify explosions; such a low value of $T_{\max }^{\prime}=2$ would appear from Fig. 6 to be an inappropriate requirement for an explosion. For that reason, the simple criterion of $T_{\max }^{\prime}>5$ for an explosion will be used tentatively below. Because $T_{\max }^{\prime}<T_{a d}^{\prime}$, such a definition requires that a system with $T_{a d}^{\prime}<5$ or $\left(\tau_{H} / \tau_{R}\right)>1 / 5$ cannot explode ex hypothesi; this is relevant when examining Figs. 1 or 3. It is worth noting that Boddington et al. [35] suggested that, in the well-mixed limit, a first-order reaction with a large activation energy cannot lead to an explosion if $\eta+1 / T_{a d}^{\prime}>1 / 4$. For $\eta \sim 0.027$ (used in this study), this condition reduces to $T_{a d}^{\prime}<\sim 4.48$, which is similar to our deduction above for a system with convection.

\subsection{Effects of timescales and physical parameters on explosive and non-explosive regimes}


Any horizontal plane defined by the axes $\left(\tau_{H} / \tau_{R}\right)$ and $\left(\tau_{H} / \tau_{C}\right)$ in Fig. 1, for a constant $\left(\tau_{H} / \tau_{D}\right)$, contains systems with convection, diffusion and reaction, in which the consumption of reactant is in principle important. In Fig. 7 , two such planes for $\mathrm{n}=1.4$ and $\left(\tau_{H} / \tau_{D}\right)=0.09$ and 0.125 are shown. The black dots show conditions for which the simulations indicated a stable reaction, i.e. $T_{\max }^{\prime}<2$. The crosses correspond to simulations for which $2<T_{\max }^{\prime}<5$ and the open circles are for conditions leading to explosion, as defined by $T_{\max }^{\prime}>5$. Lines describing approximately the boundaries $T_{\max }^{\prime} \sim 2$ and $T_{\max }^{\prime} \sim 5$ have been sketched. These boundaries diverge from each other as $\left(\tau_{H} / \tau_{R}\right)$ increases, because of the decreased sensitivity of $T_{\max }^{\prime}$ to $\left(\tau_{H} / \tau_{C}\right)$ for large $\left(\tau_{H} / \tau_{R}\right)$, as already seen in Fig. 6. Also shown in Fig. 7(a) are two experimental measurements by Archer [21], for the decomposition of azomethane. Quite importantly, these observations are in fair agreement with our numerical predictions when $T_{\max }^{\prime}>5$ is taken as the critical condition for an explosion, rather than $T_{\max }^{\prime}>2$.

Fig. 7 suggests that, as expected, for large values of $\left(\tau_{H} / \tau_{C}\right)$, i.e. vigorous mixing, the system is stable; similarly, for large $\left(\tau_{H} / \tau_{R}\right)$, i.e. relatively fast consumption of reactant (with e.g. $E$ and $q$ small), the fluid does not heat up sufficiently and accelerate the rate of reaction enough to cause an explosion. The curvature of the boundary for $T_{\max }^{\prime} \sim 5$, separating stable and unstable explosive conditions, indicates that the interaction between convection and consumption is non-linear. Such an interaction reduces the critical values of $\left(\tau_{H} / \tau_{C}\right)$ below those when each mechanism operates separately, in the presence of only diffusion. For low values of $\left(\tau_{H} / \tau_{C}\right)$, such that $\mathrm{Ra}<500$, convection is weak. Thus, when $\mathrm{Ra}<500$, the system behaves like that in the purely conductive regime with consumption of the reactant. This results in the explosion boundaries in Fig. 7 being horizontal for small Ra.

A comparison of the results in Figs. 7(a) and (b) shows that the ranges of $\left(\tau_{H} / \tau_{C}\right)$ and $\left(\tau_{H} / \tau_{R}\right)$ for which explosions occur, increase on reducing $\left(\tau_{H} / \tau_{D}\right)$. Such an increase is expected because of the weaker effect of heat conduction for the cases in Fig. 7(b), for which $\left(\tau_{H} / \tau_{D}\right)=0.09$. This means that in a weakly 
conductive system, more intense convection or faster consumption of reactant is necessary to avoid an explosion.

Fig. 8 combines Figs. 2 and 7 and presents a three-dimensional regime diagram for explosion in a spherical vessel, with the three axes showing the individual impacts of thermal conduction $\left(\tau_{H} / \tau_{D}\right)$, natural convection $\left(\tau_{H} / \tau_{C}\right)$ and the consumption of reactant $\left(\tau_{H} / \tau_{R}\right)$ on the maximum rise in temperature accompanying a reaction. These three axes form a surface separating the three-dimensional space into an explosive (inner) and a non-explosive (outer) region, where $T_{\max }^{\prime}<5$. Since all the effects represented by the axes suppress an explosion, when conditions are near the origin of Fig. 8, the gas rapidly reaches a very high temperature, and hence explodes. When any of the timescales for conduction, natural convection or chemical reaction is reduced relative to that for heating, the working point moves from the inner region towards the surface of criticality in Fig. 8, and eventually the reaction becomes stable. The relations between any two groups of timescales, with the third one fixed, can be depicted by a plane in Fig. 8. Those shown in Fig. 8 are either for $\left(\tau_{H} / \tau_{R}\right)=0$ or $\left(\tau_{H} / \tau_{C}\right)=0$ or $\left(\tau_{H} / \tau_{D}\right)=0.125$ and 0.09 . The effects of the individual chemical and physical parameters of the system can be seen clearly on each axis. Thus if the size of the reactor is increased, both $\left(\tau_{H} / \tau_{C}\right)$ and $\left(\tau_{H} / \tau_{D}\right)$ decrease $\left(\tau_{H} / \tau_{C} \propto L^{-1 / 2} ; \tau_{H} / \tau_{D} \propto L^{-2}\right)$, and the system is more prone to explode. Likewise, when the initial pressure is raised by increasing the initial concentration of reactant, the system becomes more explosive in general. However, it is essential to note that $\left(\tau_{H} / \tau_{C}\right) \propto p_{0}{ }^{1-n}$ and $\left(\tau_{H} / \tau_{D}\right) \propto p_{0}^{-n}$ (taking into account that $\kappa \propto p_{0}^{-1}$ and $\left.\rho_{0} \propto p_{0}\right)$; hence the effects of the initial pressure on the system depend on the order of the reaction [3]. When the heat of reaction $(q)$ is reduced, the values of all three ratios of timescales increase and the reaction tends to be more stable. The dotted line on the vertical plane for $\left(\tau_{H} / \tau_{C}\right)=0$ represents the predicted explosion boundary for cases with $\left(\tau_{H} / \tau_{D}\right)<0.09$. Here,the critical value of $\left(\tau_{H} / \tau_{R}\right)=1 / T_{a d}^{\prime}$ is seen to be $\sim 1 / 5 \sim 0.2$, when $\left(\tau_{H} / \tau_{C}\right)$ is close to zero, because of the criterion for an explosion, $T_{\max }^{\prime}>5$, used in this work. The entire surface of criticality could be easily constructed with further computations, but the main features have been captured by the theoretical framework and numerical results presented above. 


\section{Conclusions}

The effects of natural convection and the consumption of reactant on the explosive behaviour of an exothermic reaction, occurring inside a closed spherical vessel, have been studied with numerical simulations. It has been established that the fairly sudden transition from a relatively slow, stable reaction to a rapid, selfaccelerating explosion depends on the relative magnitudes of four timescales: that to heat up the fluid by $\Delta T_{s}$, that for natural convection, that for the conduction of heat out of the vessel and finally for chemical reaction. The main advantage of this new approach is that it separates completely the three effects inhibiting an explosion, namely thermal conduction, natural convection and the consumption of reactant. This means that whether a reaction in a closed vessel is stable or explosive can be described in a simple and intuitive manner on a three-dimensional regime diagram.

Numerical simulations were carried out for conditions with laminar flow on two planes with constant

values of $\left(\tau_{H} / \tau_{D}\right)$. It was shown that when the timescales for the consumption of reactant and natural convection are large, the reactant disappears relatively slowly and the transfer of heat out of the vessel is also slow, so the reaction produces an explosion. The new numerical results compare well with previous experimental measurements [21] for the decomposition of azomethane. All the indications are that an explosion occurs whenever $T_{\max }^{\prime}$ exceeds a value of $\sim 5$.

\section{Acknowledgements}

T-Y.L. thanks the Cambridge Overseas Trust for financial support.

\section{References}

[1] N.N. Semenov, Z. Phys., 48 (1928) 571-582.

[2] D.A. Frank-Kamenetskii, Diffusion and Heat Exchange in Chemical Kinetics (translation by J.P. Appleton), 2nd ed., Plenum Press, New York, (1969).

[3] T.-Y. Liu, A.N. Campbell, S.S.S. Cardoso, A.N. Hayhurst, Phys. Chem. Chem. Phys., 10 (2008) 5521-5530.

[4] P.L. Chambre, J. Chem. Phys., 20 (1952) 1795-1797.

[5] J.R. Parks, J. Chem. Phys., 34 (1961) 46-50.

[6] A.G. Merzhanov, Combust. Flame, 10 (1966) 341-348.

[7] T. Boddington, P. Gray, D.I. Harvey, Phil. Trans. R. Soc. London. Ser. A, 270 (1971) 467-506.

[8] B.J. Tyler, Combust. Flame, 10 (1966) 90-91.

[9] A.N. Campbell, S.S.S. Cardoso, A.N. Hayhurst, Chem. Eng. Sci., 62 (2007) 
3068-3082.

[10] S.S.S. Cardoso, P.C. Kan, K.K. Savjani, A.N. Hayhurst, J.F. Griffiths, Phys. Chem. Chem. Phys., 6 (2004) 1687-1696.

[11] S.S.S. Cardoso, P.C. Kan, K.K. Savjani, A.N. Hayhurst, J.F. Griffiths, Combust. Flame, 136 (2004) 241-245.

[12] A.G. Merzhanov, E.A. Shtessel, Astronaut. Acta, 18 (1973) 191-199.

[13] A.I. Osipov, A.V. Uvarov, N.A. Roschina, Int. J. Heat and Mass Transfer, 50 (2007) 5226-5231.

[14] P.G. Ashmore, B.J. Tyler, T.A.B. Wesley, Proc. Combust. Inst., 11 (1967) 11331140.

[15] D.R. Jones, Int. J. Heat and Mass Transfer, 16 (1) (1973) 157-167.

[16] D.R. Jones, Int. J. Heat and Mass Transfer, 17 (1) (1974) 11-21.

[17] L.-J. Sheu, J.-D. Lin, J.-R. Chen, J. Loss Prevent., 12 (2) (1999) 125- 136.

[18] A. Lazarovici, V. Volpert, J.H. Merkin, Eur. J. of Mech. B-Fluids, 24 (2) (2005) 189-203.

[19] J.S. Turner, Buoyancy effects in fluids, Cambridge University Press, Cambridge (1979).

[20] L.A. Bol'shov, P.S. Kondratenko, U.F. Strizhov, Phys. Usp., 44 (10) (2001) 9991016.

[21] W.H. Archer. Heat transfer mechanisms in exothermic reactions. Ph.D. Thesis, University of Manchester Institute of Science and Technology, 1977.

[22] P.H. Thomas, Proc. R. Soc. London. Ser. A., 262 (1961) 192-206.

[23] J. Adler, J.W. Enig, Combust. Flame, 8 (1964) 97-103.

[24] D.R. Kassoy, A. Liñan, Q. J. Mech. Appl. Math., 31 (1) (1978) 99-112.

[25] B.J. Tyler, T.A.B. Wesley, Proc. Combust. Inst., 11 (1967) 1115-1122.

[26] J.F. Griffiths, J.R. Mullins, Combust. Flame, 56 (1984) 135-148.

[27] T. Boddington, C.-G. Feng, P. Gray, Proc. R. Soc. London. Ser. A., 391 (1984) 269-294.

[28] CSIRO, Fastflo Tutorial Guide (version 3) Australia, (2000).

[29] B.J. Tyler, A.F. Tuck, Int. J. Heat and Mass Transfer, 10 (2) (1967) 251-253.

[30] O. Rice, A. Allen, H. Campbell, J. Am. Chem. Soc., 57 (1935) 2212-2222.

[31] D.A. Frank-Kamenetskii, Acta Phys.-chim. U.R.S.S., 20 (1945) 729.

[32] W. Squire, Combust. Flame, 7 (1963) 1-8.

[33] P. Gray, P.R. Lee, Combust. Flame, 9 (1965) 201-203.

[34] M. Steensma, K.R. Westerterp, Chem. Eng. Technol., 14 (1991) 147-161.

[35] T. Boddington, P. Gray, W. Kordylewski, S.K. Scott, Proc. R. Soc. London. Ser. A., 390 (1983) 13-30.

[36] M. Morbidelli, A. Varma, Chem. Eng. Sci., 43 (1) (1988) 91-102. 


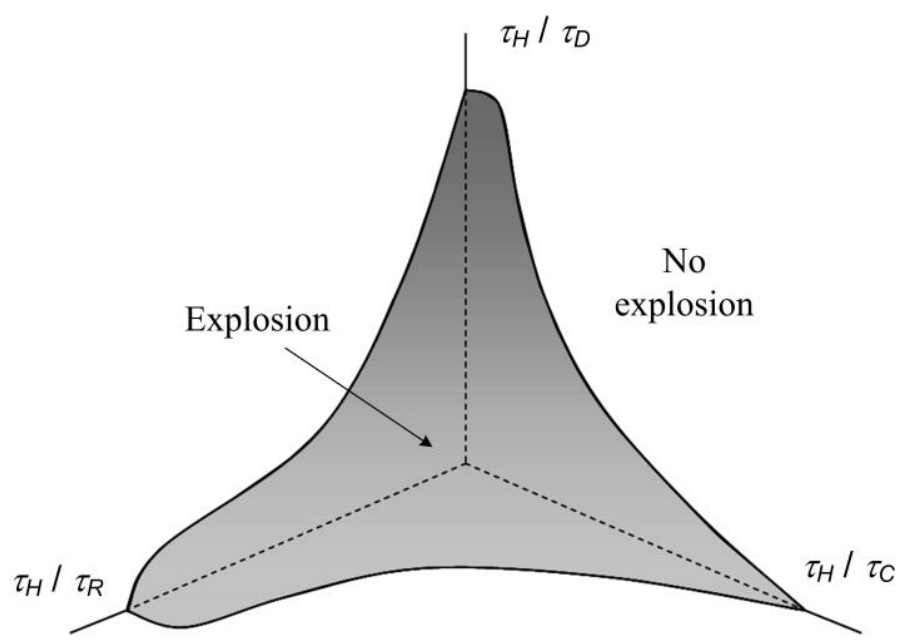

Fig. 1. Schematic regime diagram with the three axes, $\left(\tau_{H} / \tau_{C}\right),\left(\tau_{H} / \tau_{D}\right)$ and $\left(\tau_{H} / \tau_{R}\right)$, for an exothermic reaction occurring inside a closed spherical vessel. The grey surface separates the inner region, where explosions occur, from the outer one, where they do not. 


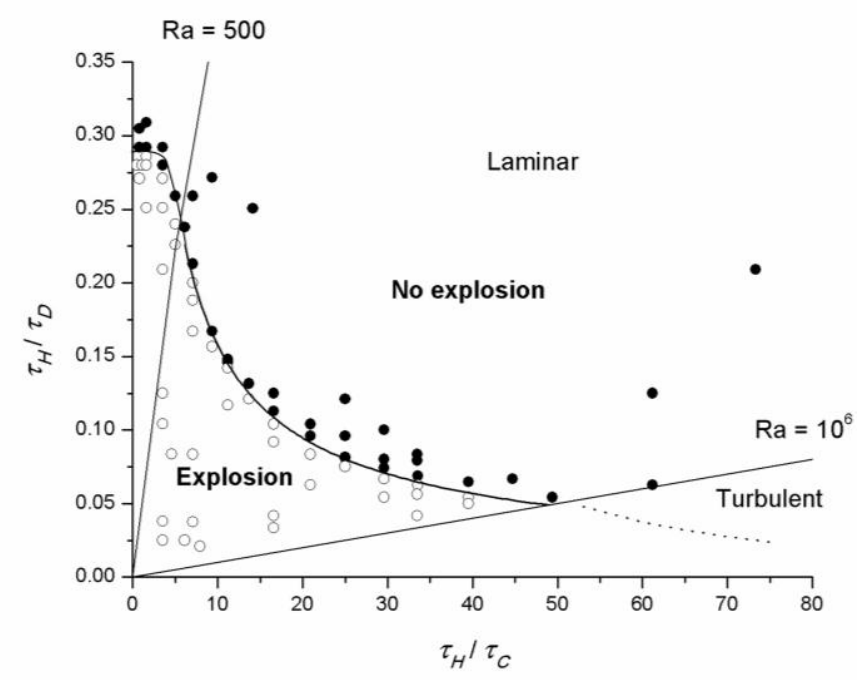

Fig. 2. The regime diagram summarising simulations [3] without consumption of reactant $\left(\tau_{H} / \tau_{R}=0\right)$, with no explosion and $\circ$ explosion, for $\eta=0.027, \operatorname{Pr}=\mathrm{Le}=1, n=1.4$ and $\gamma=1.018$. The horizontal axis denotes the well-mixed limit; the vertical axis represents the purely conductive limit. The solid and dotted lines, respectively, represent the explosion limit in the laminar and turbulent regimes.

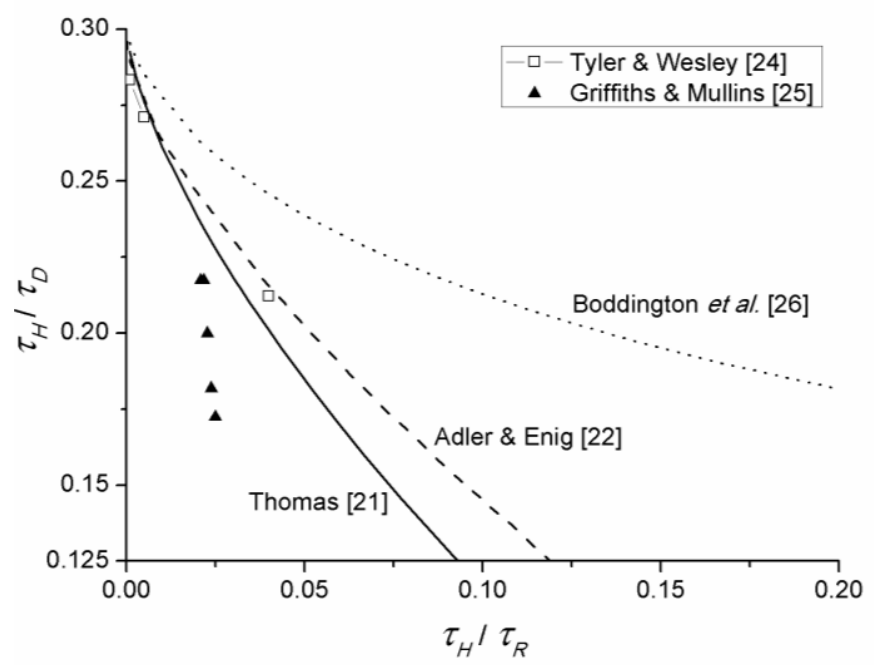


Fig. 3. Previously derived explosion limits for systems without natural convection $\left(\tau_{H} / \tau_{C}=0\right)$, expressed as a plot of $\left(\tau_{H} / \tau_{D}\right)$ versus $\left(\tau_{H} / \tau_{R}\right)$, for a first-order reaction $(n=1)$.

$\mathrm{Ra} \sim 500$
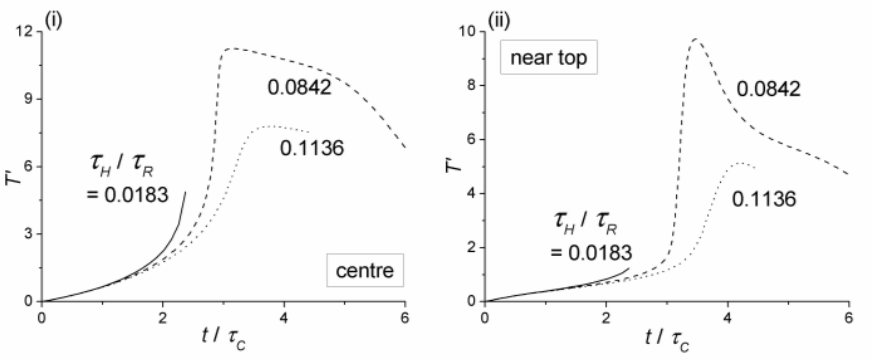

(a) Temperature
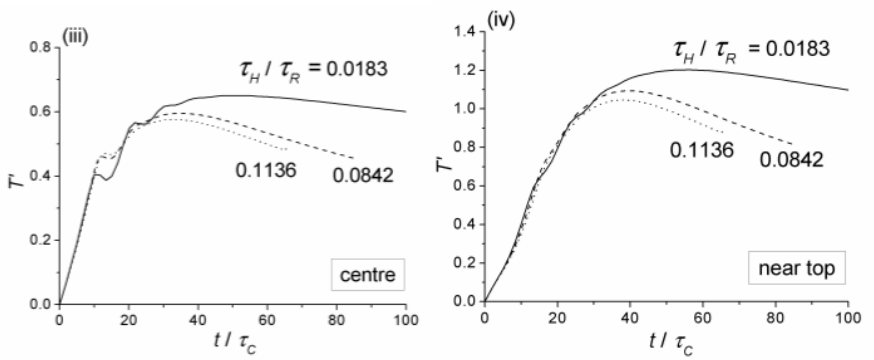

(b) Concentration
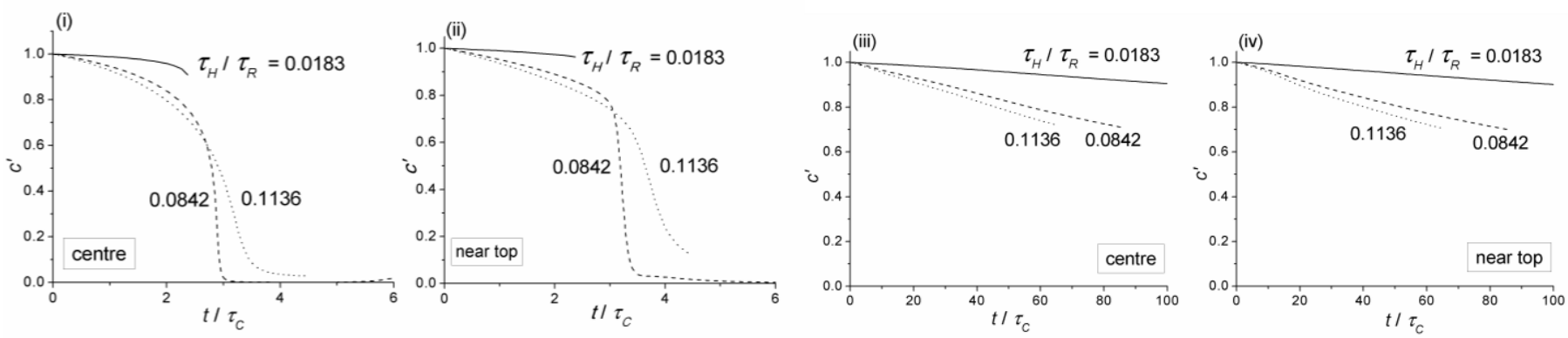

(c) Speed of flow 

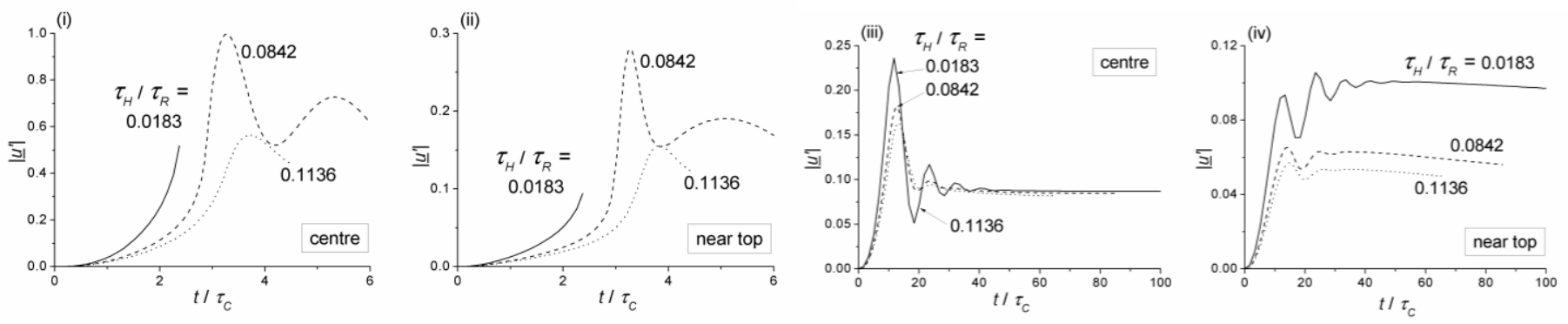

Fig. 4. Evolution of the dimensionless rise in (a) temperature, (b) dimensionless concentration of the reactant, and (c) dimensionless speed of the flow at the centre ((i) and (iii)) and at $0.8 L$ ((ii) and (iv)) vertically above the centre of the vessel for a low $\mathrm{Ra} \sim 500$ ((i) and (ii)) and a high $\mathrm{Ra} \sim 10^{5}$ ((iii) and (iv)). The three curves in each plot are for three reactions with different values of $\left(\tau_{H} / \tau_{R}\right)$, as shown, but $\left(\tau_{H} / \tau_{D}\right)=0.09$ throughout. 
(a) $\tau_{H} / \tau_{C}=2.02, \tau_{H} / \tau_{R}=0.1136, \tau_{H} / \tau_{D}=0.09, \mathrm{Ra} \sim 500$
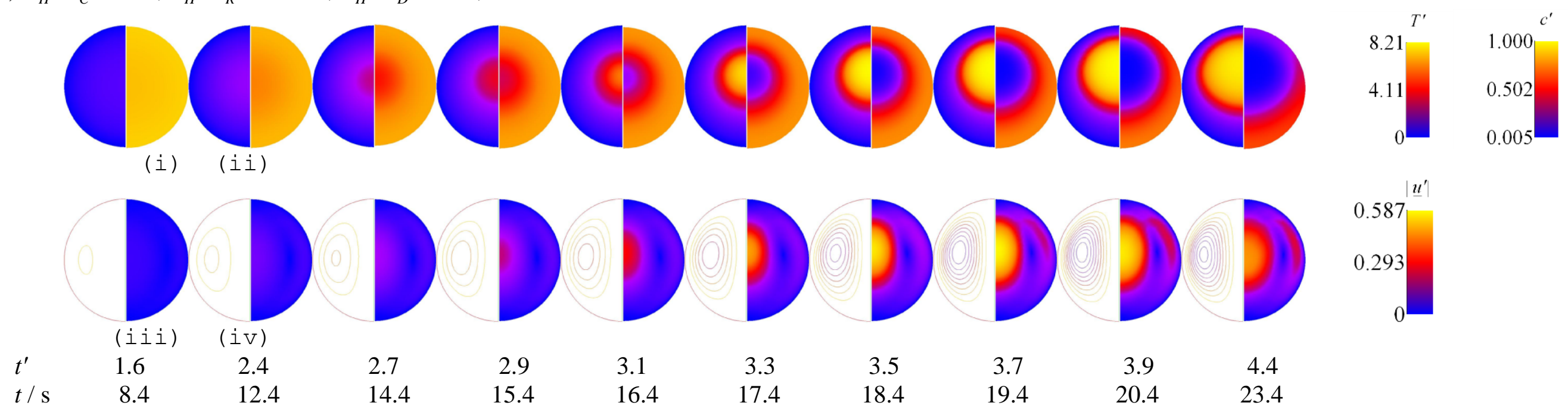

(b) $\tau_{H} / \tau_{C}=29.47, \tau_{H} / \tau_{R}=0.1136, \tau_{H} / \tau_{D}=0.09, \mathrm{Ra} \sim 10^{5}$

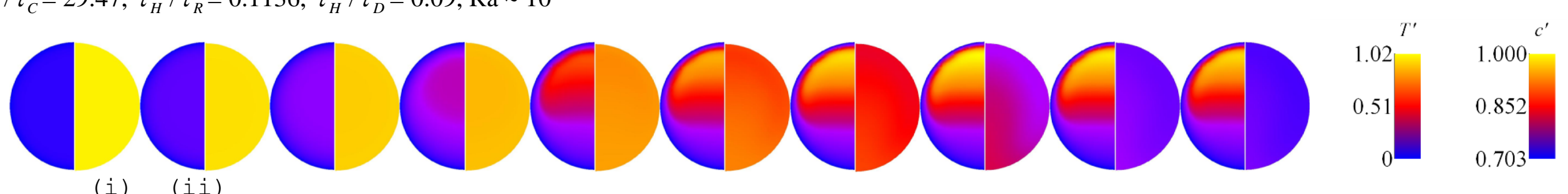

(i) (ii)
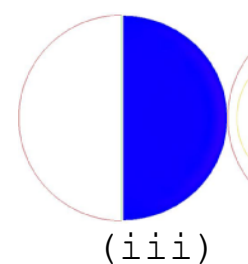

$\begin{array}{ll}t^{\prime} & 1.9\end{array}$

26.9

$t / \mathrm{s}$

9.7

0.7
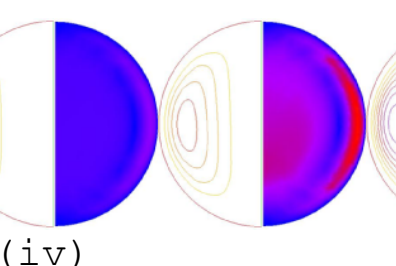

38.0

13.7
4.7

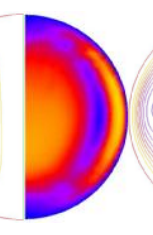

49.1

1.7

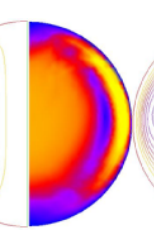

7.5

2.7

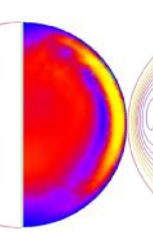

54.6
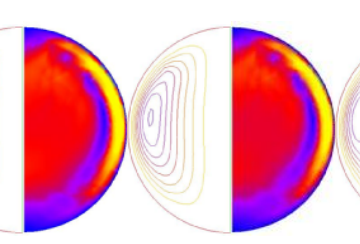

10.3

3.7
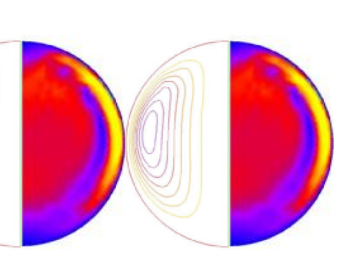

15.8

5.7
$21 \cdot 3$

7.7 


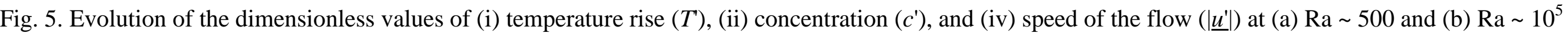
with time. The semi-circles (iii) show streamlines, with the flow being upwards close to the axis and downwards near the wall. The values of $U$ for (a) and (b) are 0.0121 and $0.1774 \mathrm{~m} \mathrm{~s}^{-1}$, respectively. The initial conditions are $T_{0}=636.2 \mathrm{~K}$ and $c_{0}=0.37 \mathrm{~mol} \mathrm{~m}^{-3}$. The colour scales for $T, c^{\prime}$, and $\left|\underline{u^{\prime}}\right|$ are shown on the right. 
(a) $\tau_{H} / \tau_{D}=0.125$

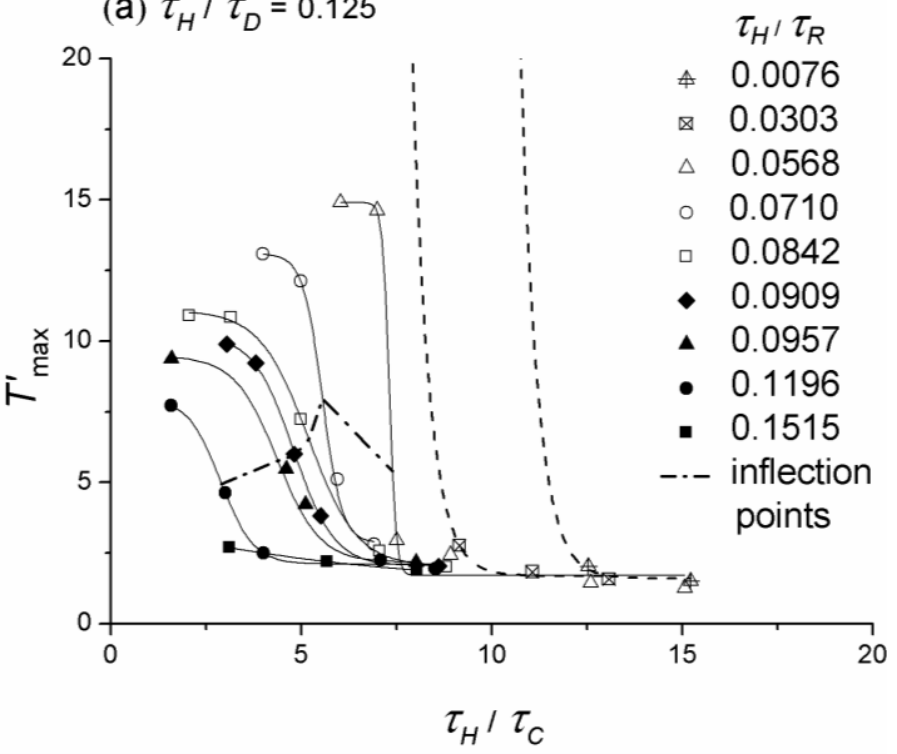

(b) $\tau_{H} / \tau_{D}=0.09$

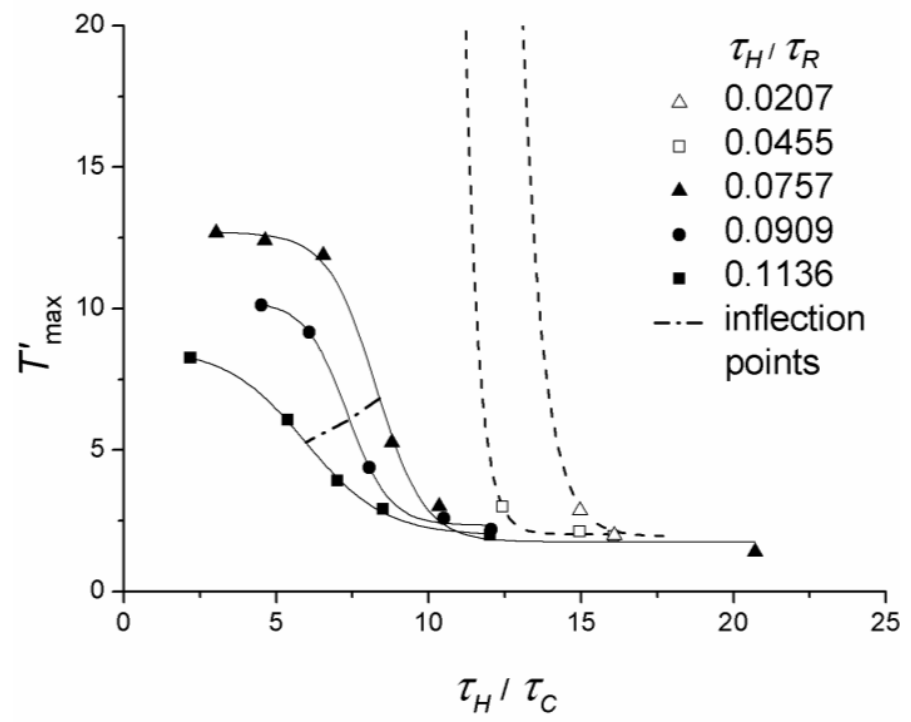

Fig. 6. Plots of $T_{\max }^{\prime}$ versus $\left(\tau_{H} / \tau_{C}\right)$ for reactions of different $\left(\tau_{H} / \tau_{R}\right)$, when $\left(\tau_{H} / \tau_{D}\right)$ is: (a) 0.125 and (b) 0.09 . For all reactions $\eta=0.027, \mathrm{Le}=\operatorname{Pr}=1, n=1.4$ and $\gamma=1.018$. The dash-dot lines connect the inflection points of the plots in both (a) and (b). In (a) it is not smooth, showing the difficulty in locating the point of inflection when $\left(\tau_{H} / \tau_{R}\right)$ is small. 
(a) $\tau_{H} / \tau_{D}=0.125$

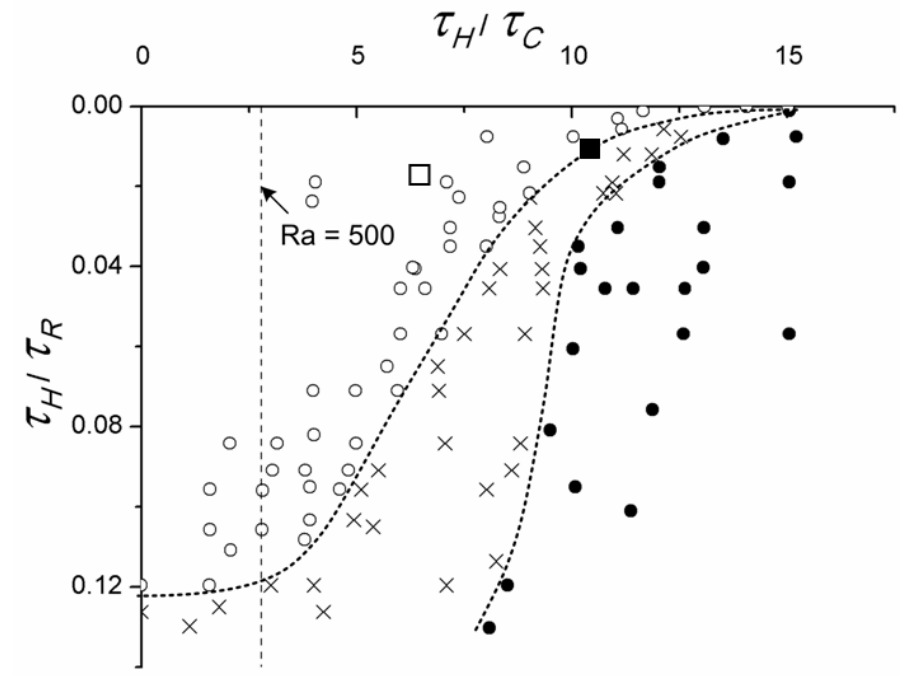

(b) $\tau_{H^{\prime}} \tau_{D}=0.09$

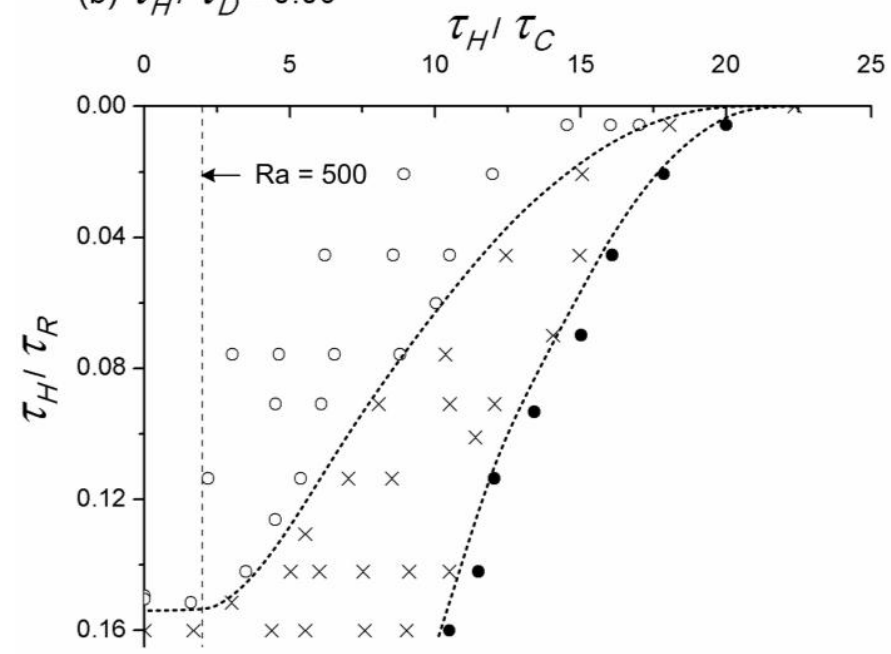


Fig. 7. Two-dimensional regime diagrams showing the effects of natural convection and consumption of reactant on the computed value of $T_{\max }^{\prime}$ for situations where thermal conduction is weak with $\left(\tau_{H} / \tau_{D}\right)=0.125$ and 0.09 , respectively. The points represent: $\circ T_{\max }^{\prime}>5 ; \times 2<T_{\max }^{\prime}<5 ; \bullet T_{\max }^{\prime}<2$. The dotted curves represent possible explosion boundaries, viz $T_{\max }^{\prime}=2$ or 5 . The simulations presented are for $\eta=0.027$, $\mathrm{Le}=\operatorname{Pr}=1, n=1.4$ and $\gamma=1.018$. Archer's [28] two experimental measurements are denoted by stable and $\square$ explosion in (a). Also, the regions where natural convection is insignificant, because $\mathrm{Ra}<500$, are shown. 


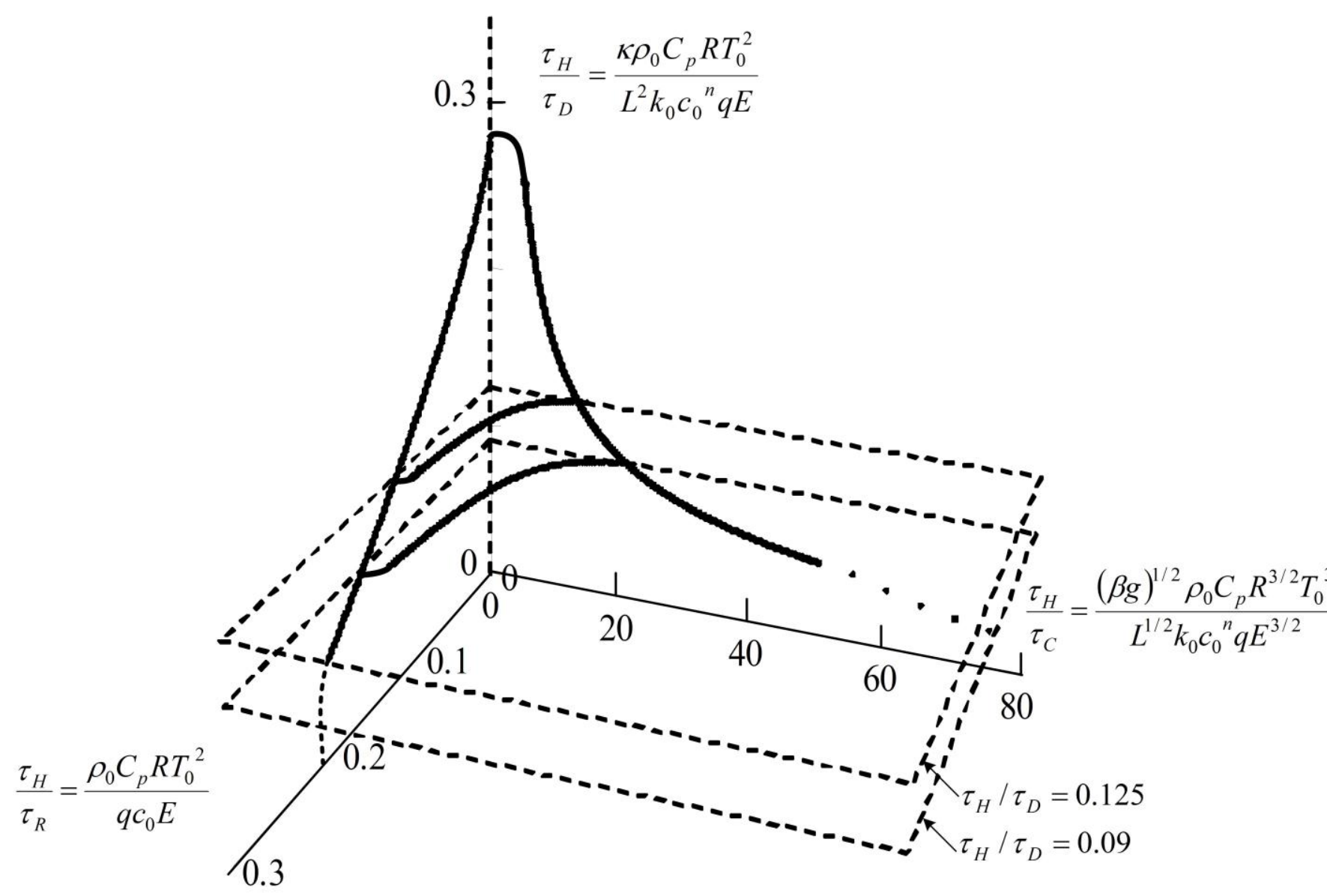

Fig. 8. Three-dimensional regime diagram for $\eta=0.027, \mathrm{Le}=\operatorname{Pr}=1, n=1.4$ and $\gamma=$ 1.018. The explosion boundaries shown on the horizontal planes defined by $\left(\tau_{H} / \tau_{D}\right)$ $=0.125$ and 0.09 are for $T_{\max }^{\prime} \sim 5$ in Figs. 7(a) and (b). The explosion boundary on the vertical plane for $\left(\tau_{H} / \tau_{R}\right)=0$ is from Fig. 2 , and the one on the plane for $\left(\tau_{H} / \tau_{C}\right)=$ 0 is similar to Fig. 3, but computed here for $n=1.4$. 